\title{
Drug discovery for breast cancer and co-instantaneous cardiovascular disease: what is the future?
}

"Therefore, the prevention and management of cardiovascular diseases for breast cancer patients is crucial and in urgent need to further promote survival and life quality, which needs to be performed not only in clinical care, but also in drug discovery."

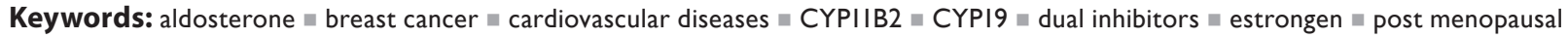

It has been estimated that approximately 1.4 million people were diagnosed with breast cancer (BC) in 2008 worldwide, which accounted for $23 \%$ of newly identified cancer cases. At the same time, 458,400 BC patients died, representing $14 \%$ of the total cancer-related deaths. On the other hand, in 2012 approximately 250,000 new BC cases will emerge in the USA; while 39,510 people will die of it [1]. This recent US cancer statistic data exhibited similar ratios to those worldwide 4 years ago, demonstrating that nowadays BC still sustains the highest incidence in females and, thus, is the top cause of cancer death. However, the mortality is actually significantly reduced with the 5-year overall survival elevating from $75 \%$ in the 1970 s to $90 \%$ after 2000 [2]. This improvement is commonly ascribed to the extensive employment of mammography, which rendered more than $60 \%$ of $\mathrm{BC}$ being diagnosed at the localized stage. More important is the system adjuvant therapy after mastectomy or breast-conserving surgery to prevent relapse. However, it has been illustrated that only approximately $40 \%$ of $\mathrm{BC}$ cases are fatal [3]. Cardiovascular diseases (CVDs), on the other hand, competed with BC as the major cause of death [4] and for the BC survivors the risks of CVDs are even higher than that of $\mathrm{BC}$ recurrence [5]. Therefore, the prevention and management of CVD for $\mathrm{BC}$ patients is crucial and in urgent need to further promote survival and life quality, which needs to be performed not only in clinical care, but also in drug discovery.

The high incidence of CVD in BC patients is not a surprise because the median age for $\mathrm{BC}$ patients to be diagnosed with BC is 61 years old and, therefore, approximately $80 \%$ of $\mathrm{BC}$ patients are older than 50 years old, and are normally postmenopausal. The increased vulnerability of postmenopausal women to CVD is demonstrated by the fact that at the same age the morbidity of CVD in postmenopausal women increased by threefold compared with the corresponding premenopausal ones. This observation also unveiled the close correlation between low estrogen levels and CVD. Estrogen reduced the apoptosis of cardiomyocytes via activating PI3K-Akt signal pathway, inhibited the growth of cardiac fibroblast and, thus, prevented ventricular hypertrophy and remodeling. The administration of estrogen also avoided heart failure postmyocardial infarction in rats and promoted survival in mice with cardiomyopathy. The shrinkage of estrogen protections due to postmenopause was therefore one of the causes of CVD in BC patients. Moreover, the comorbidity of obesity, diabetes and metabolic syndrome in this population also significantly boosted the risks of CVD. Furthermore, some cases of CVD actually resulted from the toxicities and side effects of therapies. Such treatment related CVD was found to be associated with all of the four major components of system adjuvant therapy, including endocrine hormone therapy. It has been demonstrated that radiation led to coronary artery stenosis and acute myocardial infarction with a higher incidence being observed in women that were radiated on the left side, where the heart is located, as opposed to the right side. The cardiotoxicities of chemotherapy, especially anthracyclines, trastuzumab (the monoclonal antibody interfering with the HER2) and lapatinib (dual tyrosine kinase inhibitor of HER2 and EGFR) have already been well documented with cases of left ventricular dysfunction, reduced systolic function and heart failure being reported. It is notable that the withered liver and kidney

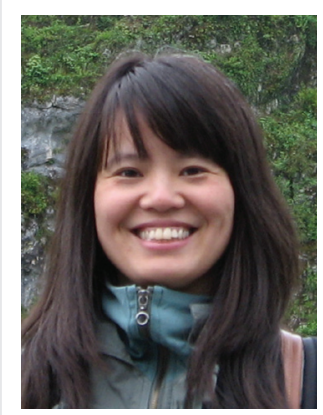

\section{Lina Yin}

Meerwiesertalweg 12, D-66123 Saarbrücken, Germany



Qingzhong $\mathbf{H u}$

Author for correspondence: Pharmaceutical \& Medicinal Chemistry, Saarland University,

Campus C2-3, D-66123

Saarbrücken, Germany

Tel.: +496830270326

E-mail: q.hu@mx.uni-saarland.de 
capacity in metabolism and excretion of these drugs, which are typical physiological features of postmenopausal BC patients, prolonged the plasma half-life and led to more exposure. Possible drug-drug interactions with the medications applied to control comorbidities such as diabetes, hypertension and metabolic syndrome also acted as complicities in exacerbating the cardiotoxicities. Regarding these problems, cardiac monitoring protocols have been proposed to implement treatments prudently including the surveillance of heart function with various imaging means and of some biomarkers such as troponin. The combinative applications with nebivolol or resveratrol, as well as new dose forms in liposomes, were also attempted to neutralize or relieve the cardiotoxicities of chemotherapy. However, the surveillance is not feasible for endocrine hormone therapy because it has to be applied for more than 5 years.

\section{"However, this also presents the challenge of achieving selectivity among CYP enzymes, especially between CYPIIB2 and CYPIIBI, which share up to $93 \%$ of homology."}

Endocrine therapy is based on the fact that estrogens stimulate the proliferation of $\mathrm{BC}$ cells that are estrogen receptors and/or progesterone receptor positive. Estrogen deprivation is, therefore, a feasible strategy to treat hormonesensitive BC, which accounts for approximately $60 \%$ of all cases. Two approaches achieving this aim have been implemented in clinic: selective estrogen receptor modulators, such as tamoxifen and raloxifen, and aromatase (CYP19) inhibitors such as letrozole and anastrozole. Selective estrogen receptor modulators compete with the natural substrates, dislodge them from binding to estrogen receptor in BC cells and, thus, inhibit the mitogenic effects stimulated by estrogen; while CYP19 inhibitors directly block the biosynthesis of estrogen by interfering with the pivotal enzyme. A comparison of clinical trials has revealed that the involvement of CYP19 inhibitors, regardless of if it is used in monotherapy or in combination with tamoxifen, reduced the recurrence risks more than application of tamoxifen alone. Since tamoxifen also exhibited severe side effects, such as endometrial cancer and cerebrovascular thrombosis, CYP19 inhibitors are commonly recommended for adjuvant therapy. However, despite the fact that CYP19 inhibitors significantly improved disease-free and relapse-free survival, the overall survival was not promoted accordingly [6]. This is probably due to the deaths that are related to side effects, especially CVD [7].

Dysfunction in lipid metabolism has been proposed as the mechanism of CYP19 inhibitorrelated ischemic CVD. However, the influence of CYP19 inhibitors on lipid metabolism was finally clarified by several clinical trials as having no negative effects a after long period of debate [8]. On the contrary, the involvement of abnormally elevated aldosterone in these pathological procedures was still unexplored. As for BC patients, the application of CYP19 inhibitors together with postmenopause status reduced estrogen to an undetectable level. The resulted estrogen deficiency not only augmented circulating aldosterone concentration directly, but also activated the whole renin-angiotensin-aldosterone system by upregulating other components, for example, renin, angiotensin converting enzyme, angiotensin II and angiotensin type 1 receptor, which led to further stimulation of aldosterone secretion [9]. Increase of potassium plasma concentration was another consequence of estrogen deficiency resulting in further enhancement of aldosterone production [10]. The abnormally high level of aldosterone retained sodium and water after its binding to mineralocorticoid receptor leading to an increase of blood volume and the subsequent chronic hypertension accompanied by hypokalemia and sometimes arrhythmia. It also boosted calcium inpouring into vascular smooth muscle cells and upregulated the expression of regulator of G-protein signaling-2 and adrenomedullin, further exacerbating chronic hypertension [11]. Furthermore, excessive aldosterone as a pro inflammation factor [12] induced the generation of reactive oxygen species [13] resulting in vascular fibrosis, vascular endothelium stiffening and finally atherosclerosis. More severe deleterious effects on the heart were mediated by inflammation and reactive oxygen species, leading to cardiac myocyte necrosis. Via stimulating collagen synthesis and fibroblast proliferation, as well as inducing the over-expression of pathological factors, such as tenascin-X, urokinase plasminogen activator receptor and a disintegrin and metalloprotease with thrombospondin motifs, redundant aldosterone eventually led to cardiac fibrosis [14]. Colluding with myocardial infarction and reperfusion injury, all 
these pathological alternations consequently rendered cardiac hypertrophy and ventricular remodeling [15]. The structural deteriorations were always accompanied with further functional degradation, such as diminished contractile capability and diastolic dysfunction, which resulted in heart failure and, ultimately, sudden death. Therefore, the control of high plasma aldosterone levels that were induced by estrogen deficiency is an effective way to cope with the cardiovascular complications in postmenopausal BC patients.

\section{"With the resolution of more and more steroidgenic CYP enzymes, comparison among them would enlighten the design into a more rational way."}

Another CYP enzyme aldosterone synthase (CYP11B2), which is affiliated to the same super family as CYP19, is the crucial enzyme in aldosterone biosynthesis and has been demonstrated to be a suitable target for the blockage of aldosterone production [16]. Since multitargeting agents exhibit advantages over combinative application of several drugs, such as improvement of patients' compliance and avoidance of possible drug-drug interactions, dual inhibitors of CYP19 and CYP11B2 are proposed as an innovative adjuvant approach in the treatment of $\mathrm{BC}[9,11]$. It is notable that the selectivities over other steroidogenic CYP enzymes are important safety criteria because of the pivotal roles of these enzymes in hormone biosynthesis: for example, $11 \beta$-hydroxylase (CYP11B1) for glucocorticoids [17] and $17 \alpha$-hydroxylase-17,20-lyase (CYP17) for androgens [18]. Despite the low homology across the CYP super family, highly conserved folding configuration and topology grant the possibility to identify dual inhibitors [19,20]; however, this also presents the challenge of achieving selectivity among CYP enzymes, especially between CYP11B2 and CYP11B1, which share up to $93 \%$ of homology. To further improve the survival of BC patients, drug-discovery efforts are needed especially for the treatment-related CVD. Besides further optimization of current drugs, the underlying mechanisms of the cardiotoxicity urgently need to be clarified to distinguish the off-target toxicity of a specific agent and the on-target side effects rooted in therapeutic mechanism. For the latter, counterwork or compensation has to be carried out with combinatory application or multitargeting agents. The mechanisms for the cardiotoxicities of cytotoxic agents. Anthracyclines are multifactorial, and most of these factors also contribute to the antitumor effects. The future is, therefore, $\operatorname{dim}$ in terms of getting rid of cardiotoxicities in this class. As for drugs targeting HER2, a primary issue that needs to be clarified is the role of HER2 in heart pathology. HER2 has been found to be important for the survival and development of cardiomyocytes with the probable involvement of neuregulin-1 [21]. The deletion of HER2 led to dilated cardiomyopathy-like chamber dilation and contractility reduction. However, the expression of HER2 in heart has been reported to be too low to mediate the cardiotoxicities of trastuzumab by direct binding. The cardiotoxicities of tyrosine kinase inhibitors, such as lapatinib, were attributed to the insufficiency of selectivity also [22]. Since current receptor tyrosine kinase inhibitors compete with ATP for the same binding site, it is extremely difficult to achieve the selectivity among numerous kinases. Nevertheless, with more effective structure-based drug design and probable detour via allosteric inhibition, more selective HER2 tyrosine kinase inhibitors are expected to decipher this primary question. On the contrary, CYP19 inhibitors are more promising to be safer after the above described dual CYP19/CYP11B2 optimization, which will also deliver better therapy to more patients (more than $60 \%$ of estrogen receptor positive versus around $25 \%$ of HER 2 positive). It is notable that the design of selective multi targeting CYP inhibitors can be inspired by the extensive experience in selective inhibitors of given steroidogenic CYP enzymes, which are significantly different from tyrosine kinase inhibitors [16-18]. This knowledge can be integrated via a ligand-based drug design approach, leading to the desired inhibitory profiles, while eluding unwanted ones. This approach has been proved to be successful by some dual inhibitors of CYP17/CYP11B1 [20] and CYP19/CYP11B2 [9,11] that were identified soon after the conception of selective multitargeting being introduced into CYP inhibitors field. Moreover, the crystals of CYP11A1, CYP19 and CYP17 have been reported; while that of CYP11B2 is also pending in the protein data bank. With the resolution of more and more steroidgenic CYP enzymes, comparison among them would enlighten the design into 
a more rational way. It is hopeful that selective dual CYP19/CYP11B2 inhibitors can be delivered from bench to bedside in the next decade and thus improve the survival and life quality of $\mathrm{BC}$ patients by avoiding the risks of CVD.

\section{Acknowledgements}

The authors would like to express their gratitude to $R W$ Hartmann for leading them into the field of steroidogenic CYP enzymes.

\section{References}

1 Siegel R, Naishadham D, Jemal A. Cancer statistics, 2012. CA Cancer J. Clin. 62(1), 10-29 (2012).

2 Siegel R, DeSantis C, Virgo K et al. Cancer treatment and survivorship statistics, 2012. CA Cancer J. Clin. 62(4), 220-241 (2012).

3 Chapman JA, Meng D, Shepherd L et al. Competing causes of death from a randomized trial of extended adjuvant endocrine therapy for breast cancer. J. Natl Cancer Inst. 100 (4), 252-260 (2008).

4 Bardia A, Arieas ET, Zhang Z et al. Comparison of breast cancer recurrence risk and cardiovascular disease incidence risk among postmenopausal women with breast cancer. Breast Cancer Res. Treat. 131(3), 907-914 (2012).

5 Patnaik JL, Byers T, DiGuiseppi C, Dabelea D, Denberg TD. Cardiovascular disease competes with breast cancer as the leading cause of death for older females diagnosed with breast cancer: a retrospective cohort study. Breast Cancer Res. 13(3), R64 (2011).

6 Amir E, Seruga B, Niraula S, Carlsson L, Ocaña A. Toxicity of adjuvant endocrine therapy in postmenopausal breast cancer patients: a systematic review and metaanalysis. J. Natl Cancer Inst. 103(17), 1299-1309 (2011).

7 Ewer MS, Glück S. A woman's heart. The impact of adjuvant endocrine therapy on cardiovascular health. Cancer 115(9), 1813-1826 (2009).

8 Younus M, Kissner M, Reich L, Wallis N. Putting the cardiovascular safety of aromatase inhibitors in patients with early breast cancer into perspective: a systematic review of the literature. Drug Saf. 34(12), 1125-1149 (2011).

$9 \quad \mathrm{Hu} \mathrm{Q}$, Yin L, Hartmann RW. Selective dual inhibitors of CYP19 and CYP11B2: targeting cardiovascular diseases hiding in the shadow of breast cancer. J. Med. Chem. 55(16), 7080-7089 (2012).

10 Ji H, Zheng W, Falconetti C, Roesch DM, Mulroney SE, Sandberg K. 17b-Estradiol deficiency reduces potassium excretion in an angiotensin type 1 receptor-dependent manner. Am. J. Physiol. Heart Circ. Physiol. 293(1), H17-H22 (2007).

11 Yin L, Hu Q, Hartmann RW. Tetrahydropyrroloquinolinone type dual inhibitors of aromatase/aldosterone synthase as a novel strategy for breast cancer patients with elevated cardiovascular risks. J. Med. Chem. 56(2), 460-470 (2013).

12 Joffe HV, Adler GK. Effect of aldosterone and mineralocorticoid receptor blockade on vascular inflammation. Heart Fail. Rev. 10(1), 31-37 (2005).

13 Fiebeler A, Luft FC. The mineralocorticoid receptor and oxidative stress. Heart Fail. Rev. 10 (1), 47-52 (2005).

14 Weber KT, Brilla CG, Campbell SE, Guarda E, Zhou G, Sriram K. Myocardial fibrosis: role of angiotensin II and aldosterone. Basic Res. Cardiol. 88(Suppl. 1), 107-124 (1993).

15 Qin W, Rudolph AE, Bond BR et al. Transgenic model of aldosterone-driven cardiac hypertrophy and heart failure. Circ. Res. 93(1), 69-76 (2003).

16 Yin L, Hu Q, Hartmann RW. 3-Pyridinyl substituted aliphatic cycles as CYP11B2
Financial \& competing interests disclosure The authors have no relevant affiliations or financial involvement with any organization or entity with a financial interest in or financial conflict with the subject matter or materials discussed in the manuscript. This includes employment, consultancies, honoraria, stock ownership or options, expert testimony, grants or patents received or pending, or royalties.

No writing assistance was utilized in the production of this manuscript. core significantly increased selectivity over CYP1A2. PLoS ONE 7(11), e48048 (2012).

17 Yin L, Lucas S, Maurer F, Kazmaier U, Hu Q, Hartmann RW. Novel imidazol-1-ylmethyl substituted 1,2,5,6-tetrahydro-pyrrolo[3,2,1ij] quinolin-4-ones as potent and selective CYP11B1 inhibitors for the treatment of Cushing's syndrome. J. Med. Chem. 55(14), 6629-6633 (2012).

$18 \mathrm{Hu}$ Q, Yin L, Jagusch C, Hille UE, Hartmann RW. Isopropylidene substitution increases activity and selectivity of biphenyl methylene 4-pyridine type CYP17 inhibitors. J. Med. Chem. 53(13), 5049-5053 (2010).

19 Abadi AH, Abou-Seri SM, Hu Q, Negri M, Hartmann RW. Synthesis and biological evaluation of imidazolylmethylacridones as cytochrome P-450 enzymes inhibitors. MedChemComm 3(6), 663-666 (2012).

$20 \mathrm{Hu}$ Q, Jagusch C, Hille UE, Haupenthal J, Hartmann RW. Replacement of imidazolyl by pyridyl in biphenyl methylenes results in selective CYP17 and dual CYP17/CYP11B1 inhibitors for the treatment of prostate cancer. J. Med. Chem. 53(15), 5749-5758 (2010).

21 Crone SA, Zhao YY, Fan L et al. ErbB2 is essential in the prevention of dilated cardiomyopathy. Nat. Med. 8(5), 459-465 (2002).

22 Hasinoff BB, Patel D. The lack of target specificity of small molecule anticancer kinase inhibitors is correlated with their ability to damage myocytes in vitro. Toxicol. Appl. Pharmacol. 249(2), 132-139 (2010). 